\title{
Functional lateralization of speech processing in adults and children who stutter
}

\section{Yutaka Sato 1,2*, Koichi Mori', Toshizo Koizumi, ${ }^{1,3}$, Yasuyo Minagawa-Kawai',4, Akihiro Tanaka ${ }^{1,5}$, Emi Ozawa ${ }^{6}$, Yoko Wakaba7 and Reiko Mazuka ${ }^{2,8}$}

1 Research Institute, National Rehabilitation Center for Persons with Disabilities, Tokorozawa, Saitama, Japan

2 Laboratory for Language Development, Brain Science Institute, RIKEN, Wako, Saitama, Japan

${ }^{3}$ Department of Otorhinolaryngology, Nara Medical University, Kashihara, Nara, Japan

${ }^{4}$ Graduate School of Human Relations, Keio University, Tokyo, Japan

${ }^{5}$ Waseda Institute for Advanced Study, Waseda University, Tokyo, Japan

${ }^{6}$ Hospital of National Rehabilitation Center for Persons with Disabilities, Tokorozawa, Saitama, Japan

7 Master's Course, Child Study Program, Graduate School of Social Welfare, Tokyo, Japan

${ }^{8}$ Department of Psychology and Neuroscience, Duke University, Durham, NC, USA

\section{Edited by:}

Judit Gervain, Université Paris

Descartes, France

\section{Reviewed by:}

Mohinish Shukla, University of

Rochester, USA

Deryk Scott Beal, Boston University, USA

\section{${ }^{*}$ Correspondence:}

Yutaka Sato, Laboratory for Language Development, Brain Science Institute, RIKEN, 2-1 Hirosawa, Wako, Saitama 351-0198, Japan.

e-mail: satoyu@brain.riken.jp
Developmental stuttering is a speech disorder in fluency characterized by repetitions, prolongations, and silent blocks, especially in the initial parts of utterances. Although their symptoms are motor related, people who stutter show abnormal patterns of cerebral hemispheric dominance in both anterior and posterior language areas. It is unknown whether the abnormal functional lateralization in the posterior language area starts during childhood or emerges as a consequence of many years of stuttering. In order to address this issue, we measured the lateralization of hemodynamic responses in the auditory cortex during auditory speech processing in adults and children who stutter, including preschoolers, with nearinfrared spectroscopy. We used the analysis-resynthesis technique to prepare two types of stimuli: (i) a phonemic contrast embedded in Japanese spoken words (/itta/ vs. /itte/) and (ii) a prosodic contrast (itta/vs. /itta?/). In the baseline blocks, only /itta/ tokens were presented. In phonemic contrast blocks, /itta/ and /itte/ tokens were presented pseudo-randomly, and /itta/ and /itta?/ tokens in prosodic contrast blocks. In adults and children who do not stutter, there was a clear left-hemispheric advantage for the phonemic contrast compared to the prosodic contrast. Adults and children who stutter, however, showed no significant difference between the two stimulus conditions. A subject-by-subject analysis revealed that not a single subject who stutters showed a left advantage in the phonemic contrast over the prosodic contrast condition. These results indicate that the functional lateralization for auditory speech processing is in disarray among those who stutter, even at preschool age. These results shed light on the neural pathophysiology of developmental stuttering.

Keywords: developmental stuttering, language development, cerebral lateralization

\section{INTRODUCTION}

Developmental stuttering is a disorder of speech fluency characterized by involuntary repetitions, prolongations, and silent blocks, especially in the initial parts of utterances. It typically starts between 2 and 6 years of age, occurring in $4-5 \%$ of all preschool children (Bloodstein, 1995; Yairi and Ambrose, 1999). Although 70-80\% of these children recover spontaneously, the stuttering persists after puberty in approximately $1 \%$ of the general population, more often in males than in females (Bloodstein, 1995; Yairi and Ambrose, 1999). Despite the previous physiological research including brain imaging techniques, the pathophysiology and the neural basis underlying developmental stuttering remains poorly understood (Brown et al., 2005; Watkins et al., 2008).

Because stuttering manifests as a motor dysfunction in speech, it has been argued that the symptoms represent breakdowns in the control, timing, and coordination of speech musculature (Brady, 1969; Bruce and Adams, 1978; Jayaram, 1984; Smith, 1995; Ludlow and Loucks, 2003). Moreover, it has been reported that persons who stutter show disrupted motor activity in the articulatory, laryngeal, and respiratory systems during speech (Zimmerman, 1980; Conture et al., 1986; Peters and Boves, 1988; Zocchi et al., 1990; Denny and Smith, 1992; Smith et al., 1993; McClean and Runyan, 2000). There is evidence, however, that linguistic demands and changes in auditory inputs affect stuttering frequency. The disfluency tends to increase when the planned utterances are long and the speech rate is altered (Jayaram, 1984; Zackheim and Conture, 2003; Blomgren and Goberman, 2008; Sawyer et al., 2008) or the syntax is complex (Gordon et al., 1986; Melnick and Conture, 2000). It has been also reported that the phonological complexity affects the speech motor dynamics in adults who stutter (Smith et al., 2010). Better fluency can be induced with changes in the auditory input, such as delayed or frequencyaltered auditory feedback of speech, choral reading (in unison with other speakers), masking by white noise, and external rhythmic cues (e.g., metronome) (Johnson and Rosen, 1937; Cherry and Sayers, 1956; van Riper, 1971; Trotter and Silverman, 1974; 
Hargrave et al., 1994; Bloodstein, 1995). These findings suggest that stuttering is not simply an impairment in the motor system. As Hampton and Weber-Fox (2008) argue, many current models of stuttering incorporate other factors: atypical neurophysiology, genetic factors, environment, personality, learning ability, auditory processing, and the ability to produce speech and language (Bloodstein, 1995; Lawrence and Barclay, 1998; Guitar, 2006).This indicates that not only a motor disability but many factors can influence stuttering.

Neuroimaging studies during the last 15 years have reported that adults who stutter demonstrate both structural and functional abnormalities compared to people who do not stutter ( $\mathrm{Wu}$ et al., 1995; Fox et al., 1996, 2000; Braun et al., 1997; Salmelin et al., 1998, 2000; De Nil et al., 2000, 2008; Ingham et al., 2000, 2004; Foundas et al., 2001, 2003; Ingham, 2001; Sommer et al., 2002; Preibisch et al., 2003; Jäncke et al., 2004; Weber-Fox et al., 2004, 2008; Biermann-Ruben et al., 2005; Brown et al., 2005; Corbera et al., 2005; Cykowski et al., 2008; Giraud et al., 2008; Watkins et al., 2008; Weber-Fox and Hampton, 2008; Chang et al., 2009; Kell et al., 2009; Sakai et al., 2009; Beal et al., 2010, 2011; Kaganovich et al., 2010; Liotti et al., 2010; Lu et al., 2010; Kikuchi et al., 2011). Among various theories of the pathophysiology of stuttering (Bloodstein, 1995; Lawrence and Barclay, 1998; Guitar, 2006), abnormal patterns of cerebral hemispheric dominance for speech processing have been consistently demonstrated. During speech production, people who stutter (PWS) show anomalous patterns characterized by overactivation, particularly in the right hemisphere, in speech-motor related brain areas, and by reduced activation in the left superior temporal, fronto-temporal, and temporo-parietal areas compared with fluency inducing conditions or fluent speakers (Wu et al., 1995; Fox et al., 1996; Braun et al., 1997; De Nil et al., 2000; Ingham et al., 2000). It has been reported that there are differences in brain responses to auditory processing during speech or passive listening tasks, and non-linguistic auditory processing between adults or children who do and do not stutter (Hampton and Weber-Fox, 2008; Beal et al., 2010, 2011; Kaganovich et al., 2010; Kikuchi et al., 2011).

Although these imaging data generally suggest that the structural and functional lateralization in language-related brain regions for speech perception or production differs between adults who do and do not stutter, the crucial question remains unresolved with regard to the possible causal relationship between stuttering and brain lateralization. Since previous studies have mainly examined adults, it has been a matter of debate whether the anatomical and functional increases in the right hemisphere in adults who stutter are the results of compensatory mechanisms used over a lifetime of stuttering (Braun et al., 1997; Preibisch et al., 2003; Chang et al., 2008; Lu et al., 2010). Alternatively, it is possible that the abnormal functional lateralization is observed in children who stutter if it is related to the onset of the stuttering. Data from children who stutter may shed light on this issue, but few such studies have been conducted (Özge et al., 2004; Chang et al., 2008; Weber-Fox et al., 2008; Kaganovich et al., 2010; Beal et al., 2011). Since stuttering typically starts prior to 6 years of age, a method that enables studying functional lateralization in this age group is necessary for elucidating the pathophysiology of developmental stuttering.
Conventional brain imaging techniques, such as functional magnetic resonance imaging (fMRI) and positron emission tomography (PET) are not well-suited for young subjects, due to safety concerns and/or the requirement for rigorous restraint. Magnetoencephalography (MEG) must be applicable for young infants for its safety, but may have difficulties to measure children younger than around 5 years. Although electroencephalography (EEG) can be applicable to young subjects, its ability to localize the focus of activity is generally poor (Minagawa-Kawai et al., 2008). In contrast, near-infrared spectroscopy (NIRS) can non-invasively measure human brain function under a variety of conditions with little restraint on young subjects, even neonates (Chen et al., 2002; Kennan et al., 2002; Peña et al., 2003; Homae et al., 2006, 2007; Minagawa-Kawai et al., 2007, 2011; Sato et al., 2010; Zaramella et al., 2001). NIRS has a reasonable resolution for exploring functional lateralization due to the limited spread of near-infrared light in the brain (Yamashita et al., 1996; Yamamoto et al. 2002; Fukui et al., 2003), unlike evoked potentials. Consequently, NIRS was our logical method of choice for examining the functional lateralization in children and adults who stutter.

We focused on cortical auditory speech processing in this study, based on its suggested involvement in stuttering (Hall and Jerger, 1978; Toscher and Rupp, 1978). Disfluency in PWS can be ameliorated by manipulating the auditory input (Johnson and Rosen, 1937; Cherry and Sayers, 1956; van Riper, 1971; Trotter and Silverman, 1974; Hargrave et al., 1994; Bloodstein, 1995), and choral reading has been shown to reverse the deactivation in the cerebral auditory regions during speech in PWS (Fox et al., 1996). While these are related to the auditory functions for self-monitoring of one's own speech, behavioral studies using a dichotic listening paradigm have demonstrated abnormal linguistic processing in PWS in terms of cerebral hemispheric dominance (Curry and Gregory, 1969; Brady and Berson, 1975; Sommers et al., 1975; Blood, 1985). Although these paradigms would be useful for examining the cerebral differences between people who do and do not stutter, young children may fail to accomplish tasks requiring overt and prompt speech responses. Similarly, tasks requiring intensive attention, like a dichotic listening test, may not be reliably performed. Thus, we used a simple listening task that is applicable to even very young subjects, and can measure functional lateralization for language processing in the absence of overt speech planning or production (Sato et al., 2003; Minagawa-Kawai et al., 2007). We used words with phonemic and prosodic contrasts as auditory stimuli. Using the same stimuli, functional brain mapping with NIRS has demonstrated a left-side advantage for the phonemic contrast compared to the prosodic contrast in normal adults, school-age, and preschool children, as well as infants older than 1 year (Furuya et al., 2001; Furuya and Mori, 2003; Sato et al., 2003).

\section{MATERIALS AND METHODS PARTICIPANTS}

Ten adults (10 males, age range 18-44 years), seven school-age children (two females and five males, age rage 6-12 years), and six preschool-age children (one female and five males, age range 3-5 years) who stutter participated in the present study. All subjects were native Japanese speakers with no reported history of hearing impairments. They did not show impairments in speech 
understanding. They were all right-handed, as assessed by the Edinburgh Handedness Inventory (Oldfield, 1971). Participants were recruited from a hospital or a self-help group for stuttering. An additional seven subjects (two adults, four school-age children, and one preschooler) were tested, but excluded from the analysis due to non-right-handedness. Stuttering severity was assessed with a 7 -scale rating system from 1 (very mild) to 7 (very severe; Akahoshi et al., 1981; Johnson et al., 1963). The stuttering severity ranged from 2 to 7 in adults $(2, n=2 ; 4, n=4 ; 5, n=2 ; 6, n=1$; $7, n=1)$, from 2 to 5 in school-age children $(2, n=2 ; 3, n=2 ; 4$, $n=2 ; 5, n=1)$, and from 2 to 4 in preschoolers $(2, n=2 ; 3, n=3 ; 4$, $n=1)$. As data of age-matched normal controls for each stuttering group, we referred to data measured in previous studies (10 normal adults, 10 males, age range $20-32$ years; 10 normal school-age children, 3 females and 7 males, age range $6-10$ years; 8 preschool-age children, 4 females and 4 males, age range 3-5 years; Furuya et al., 2001; Furuya and Mori, 2003; Sato et al., 2003). The participants or their parents gave written informed consent before the experiment. This study was approved by the Ethical Committees of National Rehabilitation Center for Persons with Disabilities (NRCD).

\section{STIMULI}

Three different inflected forms of a Japanese verb /iku/ (meaning "to go") were produced with a synthesis-by-analysis system (ASL, Kay Elemetrics Corp., USA) based on a speech signal recorded by a male adult (Imaizumi et al., 1998). By changing the formant frequencies and the vocal pitch contour, (A) past declarative /itta/ ("went"), (B) imperative /itte/ ("Go away"), and (C) interrogative /itta?/ ("went?") forms of the verb were synthesized. These words consisted of a common initial /i/ vowel with a length of $80 \mathrm{~ms}$, a 200-ms silent interval, and then the final syllable with a length of $80 \mathrm{~ms}$. Only the final syllable was changed in the two derived words. The phonemic contrasting pair /itta/ and /itte/ had different final vowels due to the manipulation of the frequencies of their formants 1 and 2 but has an identical falling pitch pattern. The prosodic contrasting pair /itta/ and /itta?/ were only different in the pitch contours of the same final vowel.

\section{NIRS RECORDING}

Recordings of the changes in hemoglobin $(\mathrm{Hb})$ concentrations in the bilateral temporal areas were made with multi-channel NIRS (ETG100, Hitachi Medical Co., Japan; OMM-2001, Shimadzu, Japan), using near-infrared lasers at two (780 and $830 \mathrm{~nm}$; ETG-100) or three wavelengths (780, 800, and $830 \mathrm{~nm}$; OMM-2001). OMM-2001 was used only for the measurement of the school-age control group. OMM-2001 used the additional middle wavelength $(800 \mathrm{~nm})$, which should have the almost identical path-length in vivo as the other wavelengths. The recording channels resided in the optical path in the brain between the nearest pairs of incident and detection probes, which were separated by $3 \mathrm{~cm}$ on the scalp surface (Nakajima et al., 1993; Fukui et al., 2003). Five incident and four detection probes arranged in a $3 \times 3$ square lattice were placed on each lateral side of the head, which made the total number of recording channels 12 on either side because each pair of adjacent incident and detection probes constituted a single measurement channel (Figure 1). After the optical measurement, the positions of the optical probes were recorded with a three-dimensional (3-D) digitizer (Polhemus,
Colchester, VT, USA). The 3-D coordinates were superimposed onto T1-weighted MR brain images for each adult subject to identify the centers of recording sites. T1-weighted anatomical images were acquired in 80 contiguous axial slices with a thickness of $2.0 \mathrm{~mm}$ using a 1.5-T scanner [Excelart, Toshiba Medical, Japan; repetition time/echo time (TR/TE) $15 / 3.4 \mathrm{~ms}$, flip angle (FA) $20^{\circ}$, matrix $256 \times 192$, field-of-view (FOV) $22 \mathrm{~cm} \times 22 \mathrm{~cm}$ ]. The channels close to the lateral end of the border between the transverse temporal gyrus and the planum temporale (PT), as projected onto a parasagittal MRI, should be in the auditory area and were referred to as auditory channels (Minagawa-Kawai et al., 2002; Furuya and Mori, 2003). This procedure selected the recording channels whose centers were within a $1.5-\mathrm{cm}$ radius of the above-mentioned border. Thus, the channels should include the signals in the auditory cortex due to the spread of the laser in the brain tissue (Yamashita et al., 1996). Since it was difficult to acquire MR brain images of some young subjects, the positions of optical probes were recorded either with a 3-D digitizer or a digital camera for identification of approximate recording locations. Because the primary auditory cortex is located approximately $6 \mathrm{~cm}$ perpendicularly above the plane containing the bilateral preauricular point (PA) and the nasion, the channels at and around the height above, and the anteroposterior position at, the PA were presumed to be in or close to the auditory area, and referred to as auditory channels.

\section{PROCEDURE}

The experiments were carried out in a sound-attenuated room. Stimuli were presented at a comfortable level (60-70 dB SPL) via insert earphones (EAR TONE 3A) for adults and a loudspeaker (i15, TANNOY) for children in accordance with the previous studies for the control subjects (Furuya et al., 2001; Furuya and Mori, 2003; Sato et al., 2003). Because it was difficult to confirm that ear plugs were tightly pushed into ears in the measurements of children, the speaker was used for them. Each participant was tested in two conditions in respective sessions with a block design paradigm. In the phonemic condition, the baseline block contained only /itta/ which was repeated

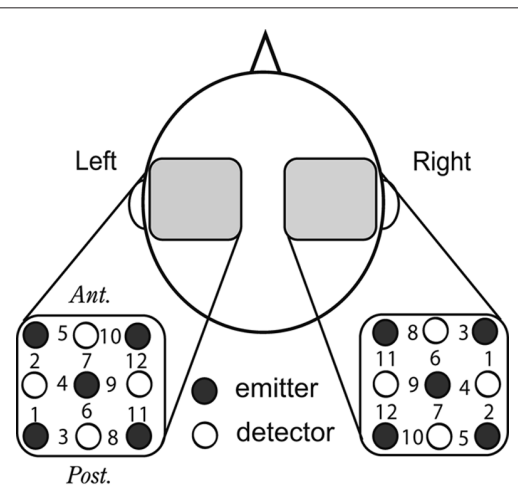

FIGURE 1 | Arrangement of NIRS recording probes. A schematic top view of the head shows that five emission and four detection probes were placed on the temporal scalp with a thermoplastic shell in a $3 \times 3$ square lattice, $3 \mathrm{~cm}$ apart from the nearest others, which constituted 12 recording sites on each side. The light incident probes are represented by filled circles; the detection probes, by open circles; measurement channels, by numerals. 
approximately once every second $(0.9-1.1 \mathrm{~s})$, whereas the test block consisted of /itta/ and /itte/ presented in a pseudo-random order with equal probabilities at the same rate as in the baseline block. Baseline and test blocks each lasted for $20 \mathrm{~s}$, and they were presented alternately at least five times. The prosodic condition was similar to the phonemic condition, except for the presentation of the /itta/ and /itta?/ combination in the test block. The presentation order of these two conditions was counterbalanced across subjects within each group.

\section{DATA PROCESSING}

Changes in the concentrations of $\mathrm{Hb}$ were calculated from the attenuation data of the laser beams. The total $\mathrm{Hb}$ responses during the test blocks in each condition were averaged synchronously and were smoothed with a 5-s moving average after manually excluding the blocks with gross motion artifacts. The maximal positive total $\mathrm{Hb}$ change was evaluated against the $10 \mathrm{~s}$ pre-test baseline period for each condition and for each auditory channel. To choose one of the auditory channels for statistical analysis, we first averaged the positive total $\mathrm{Hb}$ response across the two conditions and then selected the channel that exhibited the maximum value on each side (Minagawa-Kawai et al., 2007).

Because the measurement of $\mathrm{Hb}$ concentration obtained with continuous wave lasers lacks a reference to optical path-length, we cannot determine absolute values in principle. Consequently, the comparison or integration of data between different channels or across subjects may be difficult to justify. However, a recent study demonstrated that the optical path-lengths are similar among nearby channels and between homologous regions of left and right hemispheres within a subject (Katagiri et al., 2010). On the basis of these findings, the maximal values of total $\mathrm{Hb}$ changes in the left and right auditory channels were subjected to a two-way analysis of variance (ANOVA), with conditions (phoneme and prosody) and sides (left and right) as within-subject factors in each group. It should be noted that Katagiri et al.'s (2010) findings do not extend to a comparison across different subjects. Moreover, the two different NIRS systems that differ in the number of lasers used for calculating $\mathrm{Hb}$ concentrations (2 vs. 3 ) were used for the school-age children (ETG-100 for school-age children who stutter and OMM-2001 for school-age children who do not stutter). Since it is possible that the analyses using the $\mathrm{Hb}$ values from these machines have different sensitivities, the data were subjected to within-subject ANOVAs for each group, in which a single system was used.

In order to assess cerebral lateralization, a laterality index, $\mathrm{LI}=(L-R) /(L+R)$, was calculated from the peaks of the averaged maximal total $\mathrm{Hb}$ responses in the left $(\mathrm{L})$ and the right $(\mathrm{R})$ auditory channels. Note that LI is not affected by the possible sensitivity difference of the recording systems. LI could range from -1 to 1 , with a positive value indicating left dominance. We compared LI values between the two conditions (Wilcoxon signed-rank test) in each subject group. Subject-by-subject analysis was also performed: without averaging over repeated blocks, the left and right peaks of total $\mathrm{Hb}$ changes were collected from individual test blocks, for which respective LIs were calculated for comparison between the two contrast conditions within each subject (Mann-Whitney $U$-test).
In addition, the response peaks during the test blocks were compared with 0 (the average of the 10-s pre-test baseline) in each condition, side and group (one-sample $t$-test with false discovery rate (FDR) correction at $q<0.05$ for each group). This was done to determine whether or not the phonemic or prosodic changes in the test blocks elicited significantly larger total $\mathrm{Hb}$ changes than the baseline blocks.

\section{RESULTS \\ HEMODYNAMIC RESPONSES OF PEOPLE WHO DO AND DO NOT STUTTER}

Figure 2 shows NIRS responses in adult, school-aged, and preschoolaged PWS, in addition to typical responses of a non-stuttering adult (Furuya and Mori, 2003). This figure indicates the averaged time courses of the total $\mathrm{Hb}$ during test blocks in each condition for a non-stuttering adult and all stuttering participants. Whereas the adult who did not stutter showed a larger response on the left side under the phonemic condition and a larger response on the right side under the prosodic condition, few of the stuttering participants showed such left-right reversal between the two conditions.

Figure 3 shows the averaged peak values of the total $\mathrm{Hb}$ responses in the left and right sides under the two conditions. All groups showed significant responses during the test blocks against zero baselines on the left and the right sides under each condition (one-sample t-test, $\mathrm{p}<0.05$ respectively).

The lateralization pattern across the phonemic and prosodic conditions differed between the stuttering and control groups. Results of two-way ANOVA, with condition (phoneme and prosody) and side (left and right) as within-subject factors in each adult group showed that the PWS exhibited no significant interaction or main effects for the two factors $[F(1,9)=0.30, p>0.10 ; F(1,9)=0.02$, $p>0.10 ; F(1,9)=3.33, p>0.10$, interaction, condition, side respectively], whereas the control (Ctrl) group exhibited a significant interaction $[F(1,9)=12.07, p<0.01]$ but no effects for condition $[F(1,9)=0.32, p>0.10]$ or side $[F(1,9)=0.05, p>0.10]$, suggesting that the activations in the left and the right differed between the conditions only in the control group (Figure 3A). Simple effect tests showed that the left-side response was significantly larger than the right-side response under the phonemic condition (Holm correction, $p<0.05)$.

Similar results were observed in the school-age groups (Figure 3B). The school-age children who stutter exhibited no significant interaction or main effects for the two factors $[F(1,6)=0.81$, $p>0.10 ; F(1,6)=0.06, p>0.10 ; F(1,6)=0.11, p>0.10$, interaction, condition, side respectively]. In contrast, the school-age control group exhibited a significant interaction $[F(1,9)=12.29$, $p<0.01]$ but no effects for condition $[F(1,9)=0.00, p>0.10]$ or side $[F(1,9)=1.98, p>0.10]$. Simple effect tests showed the larger left-side response (compared to right side) under the phonemic condition $(p<0.05)$ and the larger right-side response (compared to left side) under the prosodic condition $(p<0.05)$.

The preschool children who stutter showed a different response pattern all together. They showed no significant interaction or main effect for the condition factor $[F(1,5)=0.87, p>0.10 ; F(1,5)=0.00$, $p>0.10$, interaction, condition respectively], but showed a main effect for the side factor $[F(1,5)=69.55, p<0.01]$, suggesting that right-side activation was predominant under both conditions in this 

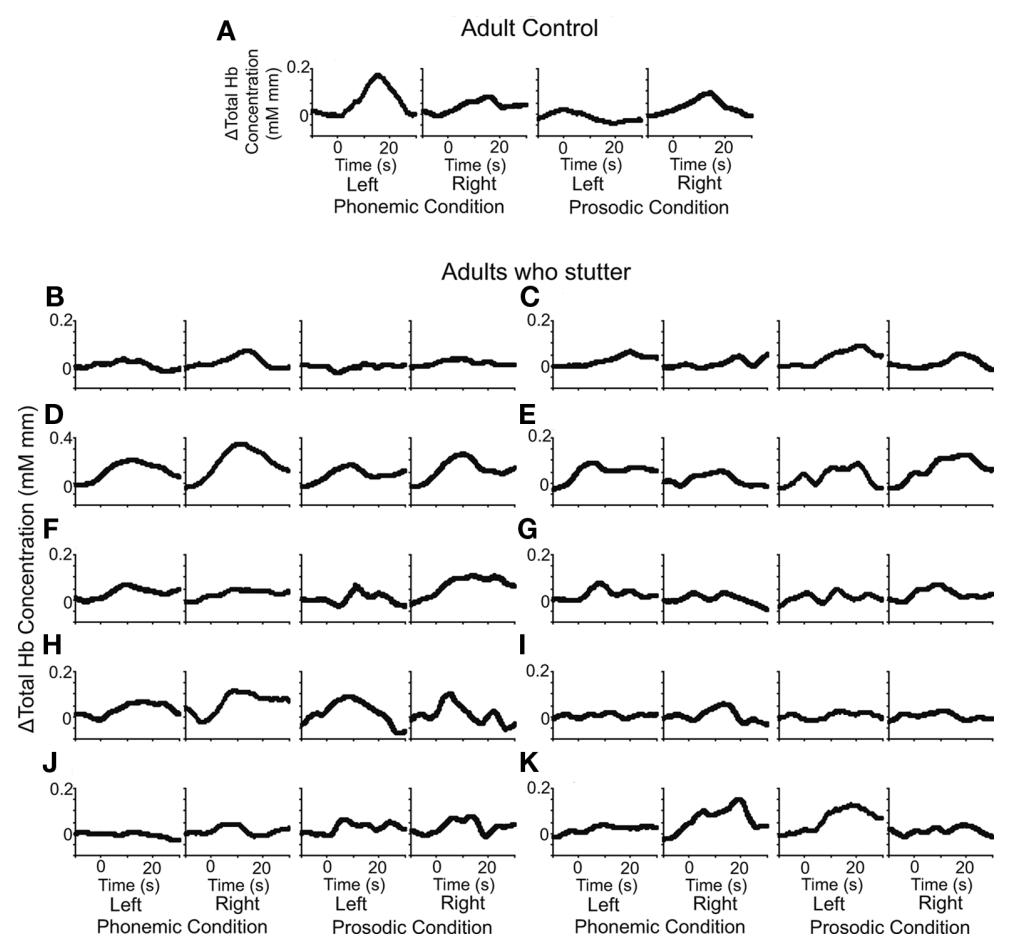

School-age children who stutter
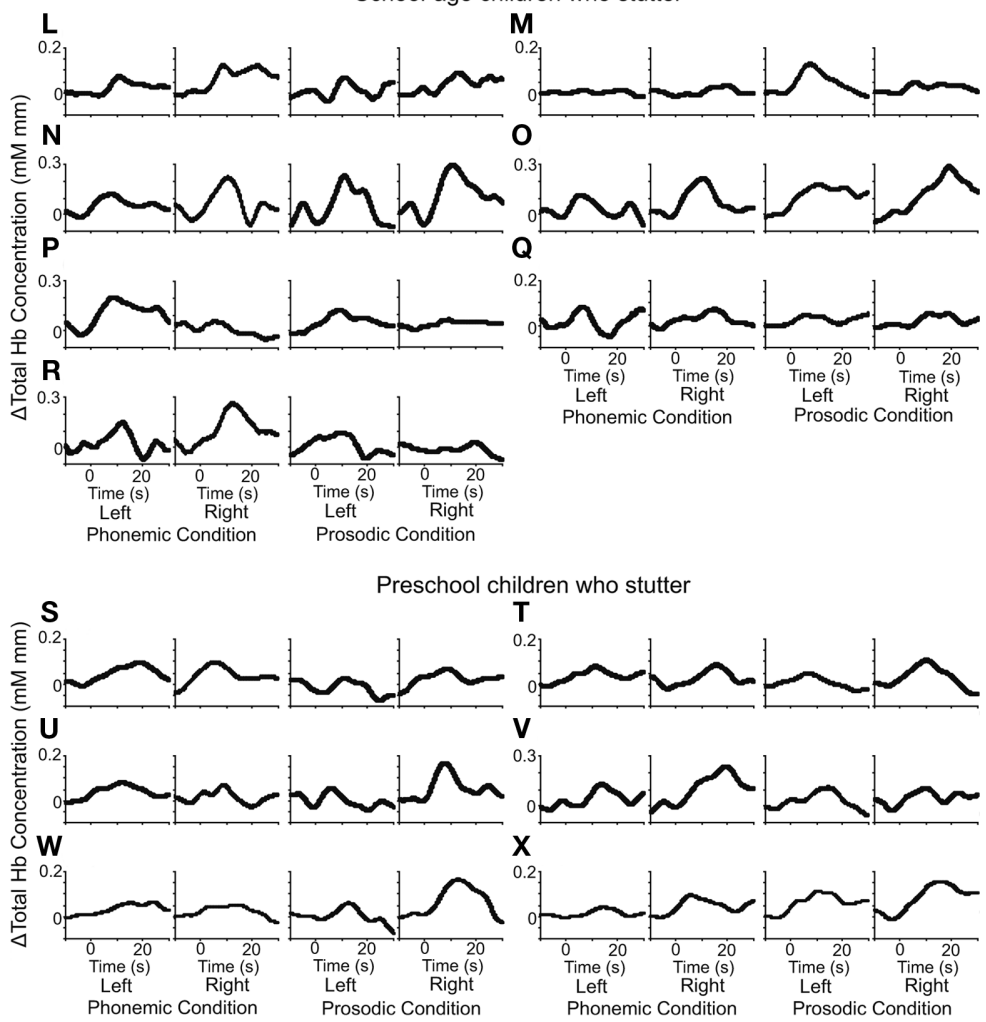

FIGURE 2 | Hemoglobin responses evoked by the phonemic and prosodic contrasts. Time courses of NIRS responses of total $\mathrm{Hb}$ in the temporal areas (left and right) are shown for individual subjects: the responses of a non-stuttering adult (adult control; data adopted from Furuya and Mori, 2003) (A), and the responses of people who stutter (PWS): adults (B-K), school-age children (L-R), and preschool children (S-X). The abscissas indicate time and the ordinates indicate total $\mathrm{Hb}$ concentration changes. The vertical lines at 0 and 20 s show the beginning and the end of the test blocks, respectively. In (A), the larger responses are seen in the left side under the phonemic condition and in the right side under the prosodic condition. Few of the stuttering subjects showed this normal response pattern under either condition. 

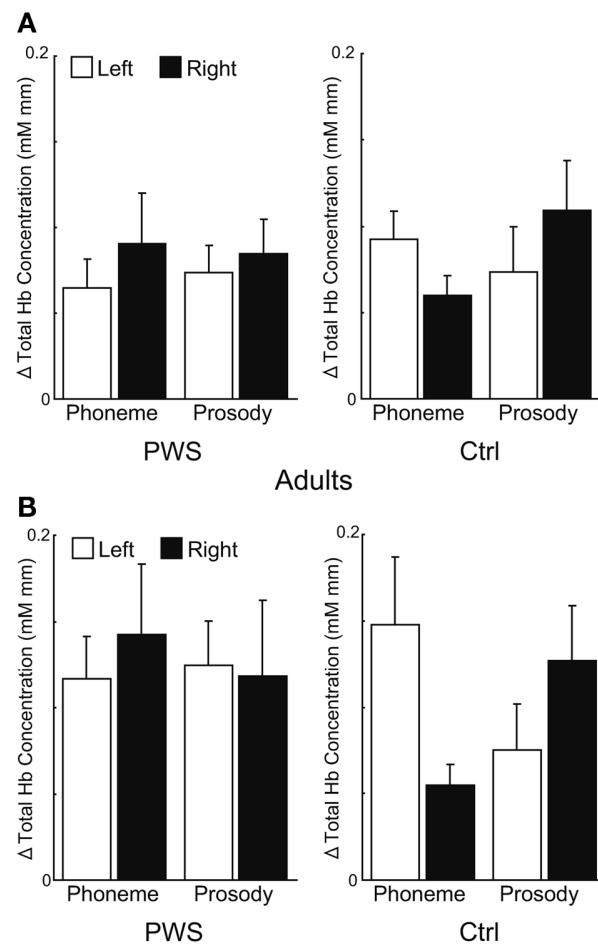

Adults

C
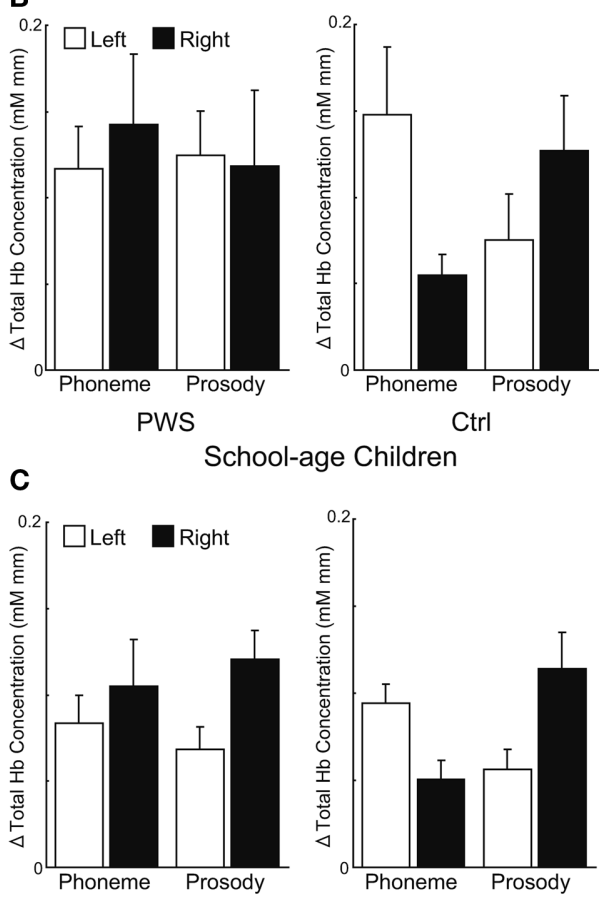

PWS

Preschool Children

FIGURE 3 | Averaged peak responses to the phonemic and prosodic contrasts. Averaged peak responses are shown according to two conditions (phoneme and prosody contrasts), people who stutter (PWS) and controls (Ctrl), and three age groups (A: adults, B: school-age children, C: preschoolers). White bars, left auditory channels; black bars, right auditory channels. Error bars: SEM. All the responses are significant. For the conditional differences, see text. Only the school-age controls were measured with OMM-2001. Control (Ctrl) data were adopted from Furuya and Mori (2003), Furuya et al. (2001), and Sato et al. (2003).

group (Figure 3C). In contrast, the preschool control group exhibited similar results as the adult and school-age control groups: they showed a significant interaction $[F(1,7)=13.32, p<0.01]$ but no effects for condition $[F(1,7)=0.80, p>0.10]$ or side $[F(1,7)=0.38$, $p>0.10]$. Simple effect tests showed the larger left-side response (compared to right side) under the phonemic condition $(p<0.05)$. Note that all three control groups showed the significant interaction of condition $\times$ side factors despite of the use of the different measurement system for the school-age control group. This must indicate that a system did not show a different sensitivity compared with the other one at least in this analysis. Moreover, similar results were observed between adults and children in either people who do or do not stutter group, suggesting that the different way of stimuli presentation, namely, by the earphones or the speaker, did not seriously impact on the current results.

\section{ANALYSES OF LATERALITY INDEX}

Figure 4 shows individual laterality indices under the phonemic and prosodic conditions in the three age groups with respective controls. First, we analyzed the differences in LIs between the two conditions in each subject group. The group analyses showed that each stuttering group failed to show significant differences in LIs between the two contrast conditions (Wilcoxon signed-rank test, adult, $p>0.10$; school-age, $p>0.10$; preschool, $p>0.10$ ). On the other hand, all control groups showed significant differences in LIs between the two contrast conditions (adult control, $p<0.01$; school-age control, $p<0.01$; preschool control, $p<0.05$ ).

\section{SUBJECT-BY-SUBJECT ANALYSIS OF LATERALITY INDEX FOR THE TWO CONDITIONS}

Subject-by-subject analysis revealed that two adults (20\%) and one school-age child (14\%) who stutter showed significant differences between their respective phonemic and prosodic LIs (white circles in Figure 4). The remaining subjects who stutter (adults 80\%; schoolage $86 \%$; preschool $100 \%$ ) showed no significant difference in LI between the two conditions (filled circles in Figure 4). Note that the significant differences of the three subjects who stutter were due to the rightward LIs for the phonemic contrast in comparison to that for the other condition. This was opposite the normal control subjects: seven of the adults (70\%), seven of the school-aged (70\%), and five of the preschool (63\%) children who do not stutter showed significant leftward LIs for the phonemic contrast. The remaining control subjects (adults 30\%; school-age 30\%; preschool 37\%) showed no significant difference in the LI between the two conditions. The ratios of subjects in the response laterality patterns differed significantly between the people who do and do not stutter in each age group (Fisher's exact test, adult, $p<0.01$; school-age, $p<0.01$; preschool, $p<0.05$ ).

\section{CORRELATION BETWEEN LATERALITY INDEX AND STUTTERING SEVERITY}

Correlation analyses indicated that stuttering severity was negatively correlated with the LI under the phonemic condition, but not significantly correlated with the LI under the prosodic condition in adults who stutter (Spearman's rho $=-0.65, p<0.05$; Spearman's rho $=0.36, p>0.05$, phonemic and prosodic conditions, respectively; Figure 5). In contrast, neither school-age nor preschool-age children who stutter showed significant correlations between severity and LI under either condition (school-age: phonemic condition, Spearman's rho $=0.26, p>0.05$; prosodic condition, Spearman's rho $=0.53, p>0.05$; preschool-age: phonemic condition, Spearman's rho $=-0.31, p>0.05$; prosodic condition, Spearman's rho $=0.19, p>0.05)$. Although we analyzed the combined data of school-age and preschool children, no significant correlation was observed between severity and LI under either condition (phonemic condition, Spearman's rho $=0.03, p>0.05$; prosodic condition, Spearman's rho $=0.45, p>0.05$ ). 


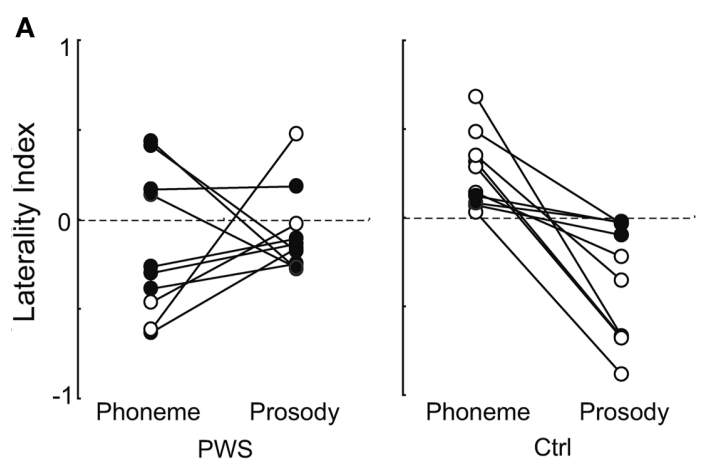

Adults
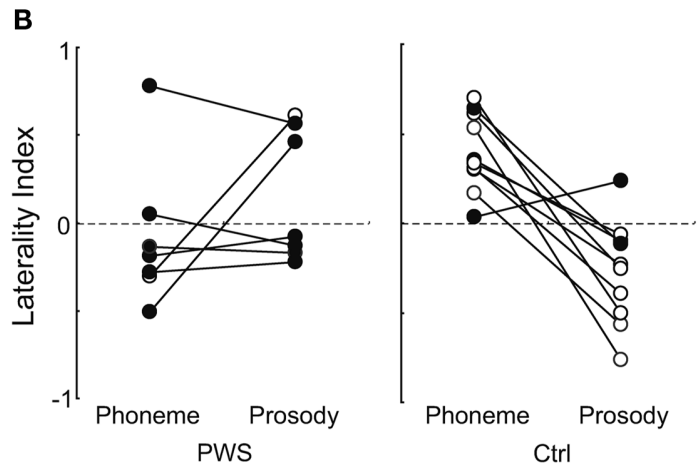

School-age Children

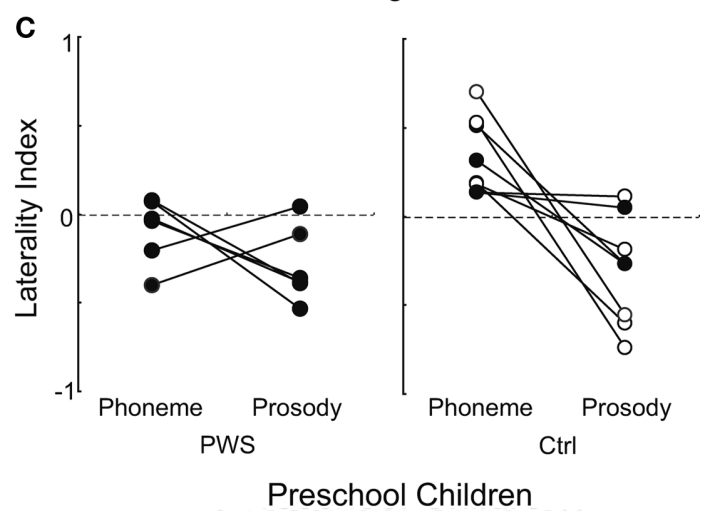

FIGURE 4 | Individual laterality indices in different groups. The individual laterality indices (circles) under the phonemic and prosodic conditions are linked with a line. The arrangement of groups and labeling convention are as in Figure 3. (A: adults, B: school-age children, C: preschoolers). Open and filled circles represent significant and non-significant differences, respectively, between the linked LIs. Note that the open circles of controls (Ctrl) show significant left-side dominance for the phonemic condition, whereas those of people who stutter (PWS) show right-side dominance for the same condition. Control (Ctrl) data were adopted from Furuya and Mori (2003), Furuya et al. (2001), and Sato et al., (2003).

\section{DISCUSSION}

To examine whether abnormal functional lateralization is associated with the onset or the result of stuttering, we used NIRS to measure brain responses of PWS during the auditory processing of phonemic and prosodic contrasts, and compared these data with age-matched control data. The $\mathrm{Hb}$ data analyses revealed that all control groups showed differential left-right activation patterns

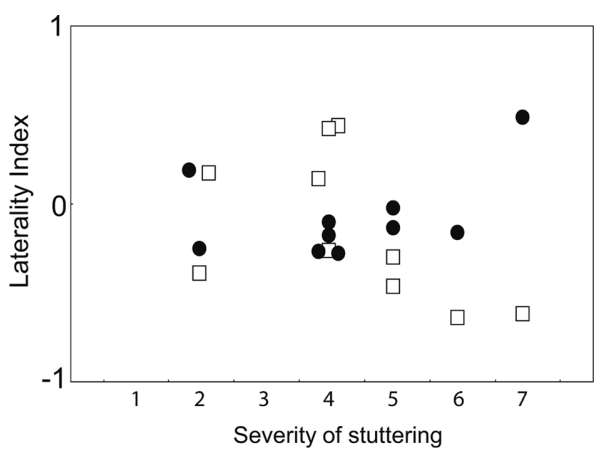

FIGURE 5 | Correlation between severity of stuttering and LIs for phonemic and prosodic conditions. The relationships of the laterality indices (ordinate) and the stuttering severity (abscissa) of adults who stutter are shown under the phonemic (white squares) and prosodic conditions (filled circles).

between the two contrast conditions. That is, normal controls showed left-side and right-side dominance for the phonemic and the prosodic contrasts, respectively, whereas the adult and schoolage stuttering groups showed no lateralized responses to either contrast. The preschool children who stutter, on the other hand, showed right-side dominance under both conditions. The group analysis of LIs found the abnormal lateralization in PWS: all control groups showed left-dominant responses to the phonemic contrast compared to the prosodic contrast, but no stuttering group showed differential lateralized responses between the two contrasts. This abnormality in functional lateralization during auditory processing is in line with previous studies using a dichotic listening paradigm (Curry and Gregory, 1969; Brady and Berson, 1975; Sommers et al., 1975; Blood, 1985) and with one using MEG (Salmelin et al., 1998). The current results in children who stutter are compatible with the previous studies showing that brain responses to auditory processing differ between children who do and do not stutter (Kaganovich et al., 2010; Beal et al., 2011). The present study confirms the abnormal auditory functional lateralization seen in PWS, and extends it from adults to down to the preschool age (i.e., shortly after the onset of stuttering). This seems to imply that it is relevant to the onset of stuttering rather than simply a consequence of long-term stuttering.

Previous studies using the same paradigm as in the current study have shown that infants around 1 year old already show a significant leftward shift of responses to phonemic contrasts compared with those to non-phonemic contrasts (Sato et al., 2003; Minagawa-Kawai et al., 2007). The present result of the atypical functional lateralization of children who stutter at 3-5 years of age still leaves room for further research to investigate how the abnormality is established. Two alternatives are possible: children who are at risk for stuttering might reset the normal lateralization they once had at around 1 year of age, or they never develop the normal pattern of lateralization before they start stuttering. Longitudinal studies of younger children of 1-3 years old are needed in order to examine the causal relationship between the abnormality and the onset of stuttering.

Adults who stutter show reduced asymmetry in PT (Foundas et al., 2001) and increased white matter volumes in the right hemisphere, including superior temporal gyrus (Jäncke et al., 2004). It 
has been also reported that PWS with atypical PT asymmetry (i. e., rightward PT asymmetry) show more disfluency than PWS with typical PT asymmetry $(L>R)$, and PWS with atypical PT asymmetry improved disfluency more with delayed auditory feedback (DAF) than those who stutter with typical PT asymmetry (Foundas et al., 2004). As alteration in the auditory signal by DAF might correct an auditory perceptual defect, it is possible that anomalous auditory processing is related to structural abnormalities in PWS. If this is true, not only anatomical PT asymmetry but anomalous functional lateralization found in this study might be clinically applicable to a prediction of improvement of disfluency by DAF. We also have to consider that brain anatomy can be changed by behavior or function. Since alterations in behavior such as music training, handedness, and language proficiency can produce changes in brain anatomy (Gaser and Schlaug, 2003; Buchel et al., 2004; Mechelli et al., 2004), most of the anatomic anomalies in PWS could be the result of either the long-term dysfunctions in auditory processing or the insufficient connection between the anterior and posterior language areas and the left motor cortex (Sommer et al., 2002; Watkins et al., 2008). In fact, no increases in volume were found in the right-hemisphere speech regions in children who stutter, and no reduction in asymmetry in the PT were observed (Chang et al., 2008). Our findings of abnormal functional lateralization as early as 3-5 years thus suggest that the anatomical abnormalities in the posterior language cortices develop as the result of more than 15 years of anomalous functional lateralization in the auditory area.

In the present study, all three age groups of PWS demonstrated similar results in terms of the lack of the stimulus dependence of LI: non-significant differences of LI were found between the phonemic and prosodic contrast conditions. Nevertheless, the $\mathrm{Hb}$ data revealed a difference in the preschool children who stutter, in contrast with the other two stuttering groups: only the preschoolers showed larger right-side responses to both phonemic and prosodic contrasts. It is possible that the affected arcuate fasciculus (Sommer et al., 2002; Chang et al., 2008) with smaller Broca's area (Chang et al., 2008) exerts a disruptive influence on the left auditory cortex and on the interaction between the left anterior and posterior language areas in the rapidly developing immature brain, thus shifting the responses to both stimuli to the right. Later, compensatory connections (yet to be specified) may restore the activation in the left auditory area to some extent especially by prosody. Alternatively, if the prosodic processing is intact in early childhood, it should be handled in the right side starting at 1 year of age (Sato et al., 2003). If the phonemic processing has to be handled by the right side due to inefficient connections between the left auditory and the anterior language areas, both phonemic and prosodic functions would be predominantly handled in the right auditory area, of which the

\section{REFERENCES}

Akahoshi, S., Ozawa, E., Kunishima, K., Suzuki, N., Doi, A., Fukawa, A., and Moriyama, H. (1981). Tentative assessment procedure for stuttering. Jpn. J. Logoped. Phoniatr. 22, 194-208.

Beal, D. S., Cheyne, D. O., Gracco, V. L., Quraan, M. A., Taylor, M. J., and De Nil, L. F. (2010). Auditory evoked fields to vocalization during passive listening and active generation in adults who stutter. Neuroimage 52, 1645-1653.

Beal, D. S., Quraan, M. A., Cheyne, D. O., Taylor, M. J., Gracco, V. L., and De Nil, L. F. (2011). Speech-induced suppression of evoked auditory fields in children who stutter. Neuroimage 54, 2994-3003.

Biermann-Ruben, K., Salmelin, R., and Schnitzler, A. (2005). Right rolandic activation during speech perception in

prosodic processing would later shift to the left to make more room for the increasing demand of phonemic processing in the right. The former is possibly less demanding for timing, so that it could be handled even with the defective arcuate fasciculus, or through the intact ventral pathway to the frontal area. The shift to the left of the prosodic processing may be related to poor perception in PWS of linguistic stimuli with prosodic information (Blood, 1996). Clearly, more longitudinal studies are needed to clarify these points.

The correlation analyses in the current study revealed that in the adults who stutter, stuttering severity was negatively correlated with the LI for the phonemic contrast, whereas neither school-age nor preschool stuttering groups showed significant correlations. This indicates that more severe stuttering symptoms are correlated with more abnormal lateralization patterns for the phonemic contrasts. This finding confirms the close relationship between the auditory deficit and stuttering. The results of the children, however, should not be directly compared to that of the adults due to the narrower distribution of stuttering severity.

To conclude, the NIRS method is practical not only for elucidating neural correlates of stuttering in children and adults alike, but also for evaluating stuttering individually. Due to the paucity of childhood functional studies, there has been long-standing controversy whether the ambiguous lateralization for cerebral linguistic processing is a cause or a result of stuttering. The current study using NIRS shows the progress of abnormal lateralization of receptive speech processing in the posterior language area, and tracks the anomaly down to the preschool age, soon after the onset of stuttering, thus narrowing down the search period for causality to between 1 year and the onset of stuttering (3-5 years). Since the NIRS method is well-suited for studying children and infants, future longitudinal studies using NIRS should greatly contribute to elucidating the pathophysiology of developmental stuttering. Subject-by-subject analysis in this study revealed that not a single subject who stutters showed a left-hemisphere advantage in the phonemic contrast processing over the prosodic contrast processing. Together with the significant correlation between abnormal lateralization in the phonemic condition and the severity of stuttering, these results may provide useful information for the clinical prognosis and treatment of stuttering.

\section{ACKNOWLEDGMENTS}

The authors would like to thank Satoshi Imaizumi for kindly providing the sound stimuli and Izumi Furuya, Ryoko Hayashi and Yoshimasa Sakata for their technical advice and contributions. This work was supported by grants from the Ministry of Health, Labor and Welfare of Japan to the second author (Koichi Mori; H14Kokoro-001, 15130801, and H16-Shogai-001).

stutterers: a MEG study. Neuroimage 25, 793-801.

Blomgren, M., and Goberman, A. M. (2008). Revisiting speech rate and utterance length manipulations in stuttering speakers. J. Commun. Disord. 41, 159-178.

Blood, G. W. (1985). Laterality differences in child stutterers: heterogeneity levels, and statistical treatments. J. Speech Hear. Res. 50, 66-72.
Blood, I. M. (1996). Disruptions in auditory and temporal processing in adults who stutter. Percept. Mot. Skills 82, 272-274.

Bloodstein, O. (1995). A Handbook on Stuttering. San Diego: Singular.

Brady, J. P. (1969). Studies on the metronome effect on stuttering. Behav. Res. Ther. 7, 197-204.

Brady, J., and Berson, J. (1975). Stuttering, dichotic listening, and cerebral 
dominance. Arch. Gen. Psychiatry 32, 1449-1452.

Braun, A. R., Varga, M., Stager, S., Schulz, G., Selbie, S., Maisog, J. M., Carson, R. E., and Ludlow, C. L. (1997). Altered patterns of cerebral activity during speech and language production in developmental stuttering. An $\mathrm{H} 2$ (15) O positron emission tomography study. Brain 120, 761-784.

Brown, S., Ingham, R. J., Ingham, J. C., Laird, A. R., and Fox, P. T. (2005). Stuttered and fluent speech production: an ALE meta-analysis of functional neuroimaging studies. Hum. Brain Mapp. 25, 105-117.

Bruce, M. C., and Adams, M. R. (1978). Effects of two types of motor practice on stuttering adaptation. J. Speech Hear. Res. 21, 421-428.

Buchel, C., Raedler, T., Sommer, M., Sach, M., Weiller, C., and Koch, M. A. (2004). White matter asymmetry in the human brain: a diffusion tensor MRI study. Cereb. Cortex 14, 945-951.

Chang, S. E., Erickson, K. I., Ambrose, N. G., Hasegawa-Johnson, M. A., and Ludlow, C. L. (2008). Brain anatomy differences in childhood stuttering. Neuroimage 39, 1333-1344.

Chang, S. E., Kenney, M. K., Loucks, T.M., and Ludlow, C. L. (2009). Brain activation abnormalities during speech and non-speech in stuttering speakers. Neuroimage 46, 201-212.

Chen, S., Sakatani, K., Lichty, W., Ning, P., Zhao, S., and Zuo, H. (2002). Auditory-evoked cerebral oxygenation changes in hypoxic-ischemic encephalopathy of newborn infants monitored by near infrared spectroscopy. Early Hum. Dev. 67, 113-121.

Cherry, C., and Sayers, B. (1956). Experiments upon the total inhibition of stammering by external control, and some clinical results. J. Psychosom. Res. 1, 233-246.

Conture, E. G., Rothenberg, M., and Molitor, R. D. (1986). Electroglottographic observations of young stutterers' fluency. J. Speech Hear. Res. 29, 384-393

Corbera, S., Corral, M. J., Escera, C., and Idiazábal, M. A. (2005). Abnormal speech sound representation in persistent developmental stuttering. Neurology 65, 1246-1252.

Curry, F. K.W., and Gregory, H. H. (1969). The performance of stutterers on dichotic listening tasks thought to reflect cerebral dominance. J. Speech Hear. Res. 12, 73-82.

Cykowski, M., Kochunov, P., Ingham, R. J., Ingham, J.C., Mangin, J. F., Rivière, D., Lancaster, J. L., and Fox, P. T. (2008). Perisylvian sulcal morphology and cerebral asymmetry patterns in adults who stutter. Cereb. Cortex 18,571-583.
De Nil, L. F., Beal, D. S., Lafaille, S. J., Kroll, R. M., Crawley, A. P., and Gracco, V. L. (2008). The effects of simulated stuttering and prolonged speech on the neural activation patterns of stuttering and nonstuttering adults. Brain Lang. 107, 114-123.

De Nil, L. F., Kroll, R. M., Kapur, S., and Houle, S. (2000). A positron emission tomography study of silent and oral single word reading in stuttering and nonstuttering adults. J. Speech Hear. Res. 43, 1038-1053.

Denny, M., and Smith, A. (1992). Gradations in a pattern of neuromuscular activity associated with stuttering. J. Speech Hear. Res. 35, 1216-1229.

Foundas, A. L., Bollich, A. M., Corey, D. M., Hurley, M., and Heilman, K. M. (2001). Anomalous anatomy of speech-language areas in adults with persistent developmental stuttering. Neurology 57, 207-215.

Foundas, A. L., Bollich, A. M., Feldman, J., Corey, D. M., Hurley, M., Lemen, L. C., and Heilman, K. M. (2004). Aberrant auditory processing and atypical planum temporale in developmental stuttering. Neurology 63, 1640-1646.

Foundas, A. L., Corey, D. M., Angeles, V., Bollich, A. M., Crabtree-Hartman, E., and Heilman, K. M. (2003). Atypical cerebral laterality in adults with persistent developmental stuttering. Neurology 61, 1378-1385.

Fox, P. T., Ingham, R. J., Ingham, J. C., Hirsch, T. B., Downs, J. H., Martin, C., Jerabek, P., Glass, T., and Lancaster, J. L. (1996). A PET study of the neural systems of stuttering. Nature 382 , 158-161.

Fox, P. T., Ingham, R. J., Ingham, J. C., Zamarripa, F., Xiong, J. H., and Lancaster, J. L. (2000). Brain correlates of stuttering and syllable production. A PET performance-correlation analysis. Brain 123, 1985-2004.

Fukui, Y., Ajichi, Y., and Okada, E. (2003). Monte Carlo prediction of near-infrared light propagation in realistic adult and neonatal head models. Appl. Opt. 42, 2881-2887.

Furuya, I., and Mori, K. (2003). Cerebral lateralization in spoken language processing measured by multichannel near-infrared spectroscopy (NIRS). Brain Nerve 55, 226-231.

Furuya, I., Mori, K., Minagawa, Y., and Hayashi, R. (2001). Cerebral lateralization of speech processing in infants measured by near-infrared spectroscopy. Neurosci. Res. S25, S125.

Gaser, C., and Schlaug, G. (2003). Gray matter differences between musicians and nonmusicians. Ann. N. Y. Acad. Sci. 999, 514-517.

Giraud, A. L., Neumann, K., BachoudLevi, A. C., von Gudenberg, A. W., Euler, H. A., Lanfermann, H., and
Preibisch, C. (2008). Severity of dysfluency correlates with basal ganglia activity in persistent developmental stuttering. Brain Lang. 104 190-199.

Gordon, P., Luper, H. L., and Peterson, H. A. (1986). The effects of syntactic complexity on the occurrence of disfluencies in 5 year old nonstutterers. J. Fluency Disord. 11, 151-164.

Guitar, B. (2006). Stuttering: An Integrated Approach to Its Nature and Treatment. Philadelphia: Lippincott Williams \& Wilkins.

Hall, J. W., and Jerger, J. (1978). Central auditory function in stutterers. $J$. Speech Hear. Res. 21, 324-337.

Hampton, A., and Weber-Fox, C. (2008). Non-linguistic auditory processing in stuttering: evidence from behavior and event-related brain potentials. J. Fluency Disord. 33, 253-273.

Hargrave, S., Kalinowski, J., Stuart, A., Armson, J., and Jones, K. (1994). Effect of frequency-altered feedback on stuttering frequency at normal and fast speech rates. J. Speech Hear. Res. 37, 1313-1319.

Homae, F., Watanabe, H., Nakano, T., Asakawa, K., and Taga, G. (2006). The right hemisphere of sleeping infant perceives sentential prosody. Neurosci. Res. 54, 276-280.

Homae, F., Watanabe, H., Nakano, T., and Taga, G. (2007). Prosodic processing in the developing brain. Neurosci. Res. 59, 29-39.

Imaizumi, S., Mori, K., Kiritani, S., Hosoi, H., and Tonoike, M. (1998). Taskdependent laterality for cue decoding during spoken language processing. Neuroreport 9, 899-903.

Ingham, R. J. (2001). Brain imaging studies of developmental stuttering. J. Commun. Disord. 34, 493-516.

Ingham, R. J., Fox, P. T., Ingham, J. C., Xiong, J. H., Zamarripa, F., Hardies, L. J., and Lancaster, J. L. (2004). Brain correlates of stuttering and syllable production: gender comparison and replication. J. Speech Hear. Res. 47, 321-341.

Ingham, R. J., Fox, P. T., Ingham, J. C., and Zamarripa, F. (2000). Is overt stuttered speech a prerequisite for the neural activations associated with chronic developmental stuttering? Brain Lang. 75, 163-194.

Jäncke, L., Hänggi, J., and Steinmetz, H. (2004). Morphological brain differences between adult stutterers and non-stutterers. BMC Neurol. 4, 23 doi: 10.1186/1471-2377-4-23

Jayaram, M. (1984). Distribution of stuttering in sentences: relationship to sentence length and clause position. $J$. Speech Hear. Res. 27, 338-341.

Johnson, W., Darley, F. L., and Spriestersbach,D.C.(1963). Diagnostic
Methods in Speech Pathology. New York: Harper \& Row.

Johnson, W., and Rosen, L. (1937). Studies in the psychology of stuttering: VII Effect of certain changes in speech pattern upon frequency of stuttering. J. Speech Disord. 2, 105-109.

Kaganovich, N., Wray, A. H., and WeberFox, C. (2010). Non-linguistic auditory processing and working memory update in pre-school children who stutter: an electrophysiological study. Dev. Neuropsychol. 35, 712-736.

Katagiri, A., Dan, I., Tuzuki, D., Okamoto, M., Yokose, N., Igarashi, K., Hoshino, T. Fujiwara, T., Katayama, Y., Yamaguchi, Y., and Sakatani, K. (2010). Mapping of optical pathlength of human adult head at multi-wavelengths in near infrared spectroscopy. Adv. Exp. Med. Biol. 662, 205-212.

Kell, C. A., Neumann, K., von Kriegstein, K., Posenenske, C., von Gudenberg, A. W., Euler, H., and Giraud, A. L. (2009). How the brain repairs stuttering. Brain 132, 2747-2760.

Kennan, R. P., Kim, D., Maki, A., Koizumi, H., and Constable, R. T. (2002). Noninvasive assessment of language lateralization by transcranial near infrared optical topography and functional MRI. Hum. Brain Mapp. 16, 183-189.

Kikuchi, Y., Ogata, K., Umesaki, T., Yoshiura, T., Kenjo, M., Hirano, Y., Okamoto, T., Komune, S., and Tobimatsu, S. (2011). Spatiotemporal signatures of an abnormal auditory system in stuttering. Neuroimage 55, 891-899.

Lawrence, M., and Barclay, D. M. III. (1998). Stuttering: a brief review. Am. Fam. Physician 57, 2175-2178.

Liotti, M., Ingham, J.C., Takai, O., Paskos, D. K., Perez, R., and Ingham, R. J. (2010). Spatiotemporal dynamics of speech sound perception in chronic developmental stuttering. Brain Lang. 115, 141-147.

Lu, C., Peng, D., Chen, C., Ning, N. Ding, G., Li, K., Yang, Y., and Lin, C. (2010). Altered effective connectivity and anomalous anatomy in the basal ganglia-thalamocortical circuit of stuttering speakers. Cortex 46, 49-67.

Ludlow, C. L., and Loucks, T. (2003). Stuttering: a dynamic motor control disorder. J. Fluency Disord. 28, 273-295.

McClean, M. D., and Runyan, C. M. (2000). Variations in the relative speeds of orofacial structures with stuttering severity. J. Speech Hear. Res. 43, 1524-1531.

Mechelli, A., Crinion, J. T., Noppeney, U., O'Doherty, J., Ashburner, J. Frackowiak, R. S., and Price, C. J. (2004). Neurolinguistics: structural plasticity in the bilingual brain. Nature 431,757 . 
Melnick, K. S., and Conture, E. G. (2000). Relationship of length and grammatical complexity to the systematic and nonsystematic speech errors and stuttering of children who stutter. J. Fluency Disord. 25, 21-45.

Minagawa-Kawai, Y., Mori, K., Furuya, I., Hayashi, R., and Sato, Y. (2002). Assessing cerebral representations of short and long vowel categories by NIRS. Neuroreport 13, 581-584.

Minagawa-Kawai, Y., Mori, K., Hebden, J. C., and Dupoux, E. (2008). Optical imaging of infants' neurocognitive development: recent advances and perspectives. Dev. Neurobiol. 68, 712-728.

Minagawa-Kawai, Y., Mori, K., Naoi, N., and Kojima, S. (2007). Neural attunement processes in infants during the acquisition of a language-specific phonemic contrast. J. Neurosci. 27, 315-321.

Minagawa-Kawai, Y., van der Lely, H., Ramus, F., Sato, Y., Mazuka, R., and Dupoux, E. (2011). Optical brain imaging reveals general auditory and language-specific processing in early infant development. Cereb. Cortex 21, 254-261.

Nakajima, T., Miki, A., Kuwabara, T., Watanabe, H., Shimizu, K., Takeo, K., Iwamoto, S., Fujita, M., Naruse, S., and Yuasa, T. (1993). Functional mapping of the human visual system with near-infrared spectroscopy and BOLD functional MRI. J. Appl. Physiol. $75,1842-1846$.

Oldfield, R.C. (1971). The assessment and analysis of handedness: the Edinburgh inventory. Neuropsychologia 9, 97-113.

Özge, A., Toros, F., and Cömeleko lu, U. (2004). The role of hemispheral asymmetry and regional activity of quantitative EEG in children with stuttering. Child Psychiatry Hum. Dev. 34, 269-280.

Peña, M., Maki,A., Kovacic, D., DehaeneLambertz, G., Koizumi, H., Bouquet, F., and Mehler, J. (2003). Sounds and silence: an optical topography study of language recognition at birth. Proc. Natl. Acad. Sci. U.S.A. 103, 11702-11705.

Peters, H. F. M., and Boves, L. (1988). Coordination of aerodynamic and phonatory processes in fluent speech of stutterers. J. Speech Hear. Res. 31, 352-361.
Preibisch, C., Neumann, K., Raab, P., Euler, H. A., von Gudenberg, A. W., Lanfermann, H., and Giraud, A. L. (2003). Evidence for compensation for stuttering by the right frontal operculum. Neuroimage 20, 1356-1364.

Sakai, N., Masuda, S., Shimotomai, T., and Mori, K. (2009). Brain activation in adults who stutter under delayed auditory feedback: an fMRI study. Int . J. Speech Lang. Pathol. 11, 2-11.

Salmelin, R., Schnitzler, A., Schmitz, F., and Freund, H. J. (2000). Single word reading in developmental stutterers and fluent speakers. Brain 123, 1184-1202.

Salmelin, R., Schnitzler, A., Schmitz, F., Jäncke, L., Witte, O. W., and Freund, H. J. (1998). Functional organization of the auditory cortex is different in stutterers and fluent speakers. Neuroreport 9, 2225-2229.

Sato, Y., Mori, K., Furuya, I., Hayashi, R., Minagawa-Kawai, Y., and Koizumi, T. (2003). Developmental changes in cerebral lateralization to spoken language in infants: measured by near-infrared spectroscopy. Jpn. J. Logoped. Phoniatr. 44, 165-171.

Sato, Y., Sogabe, Y., and Mazuka, R. (2010). Development of hemispheric specialization for lexical pitch-accent in Japanese infants. J. Cogn. Neurosci. 22, 2503-2513.

Sawyer, J., Chon, H., and Ambrose, N. G. (2008). Influences of rate, length, and complexity on speech disfluency in a single-speech sample in preschool children who stutter. J. Fluency Disord. 33, 220-240.

Smith, A. (1995). "Muscle activity in stuttering," in Proceedings of the 1st World Congress of Fluency Disorders, eds C. W. Starkweather and H. F. M Peters (Nijmegen: Nijmegen University Press), 39-42.

Smith, A., Luschei, E., Denny, M., Wood, J., Hirano, M., and Badylak, S. (1993). Spectral analyses of laryngeal and orofacial muscles in stutterers. J. Neurol. Neurosurg. Psychol. 56, 1301-1311.

Smith, A., Sadagopan, N., Walsh, B., and Weber-Fox, C. (2010). Increasing phonological complexity reveals heightened instability in inter-articulatory coordination in adults who stutter. J. Fluency Disord. 35, 1-18.
Sommer, M., Koch, M. A., Paulus, W. Weiller, C., and Büchel, C. (2002). Disconnection of speech-relevant brain areas in persistent developmental stuttering. Lancet 360, 380-383.

Sommers, R. K., Brady, W.A., and Moore, W. J. Jr. (1975). Dichotic ear preferences of stuttering children and adults. Percept. Mot. Skills 41, 931-938.

Toscher, M. M., and Rupp, R. R. (1978). A study of the central auditory processes in stutterers using the Synthetic Sentence Identification (SSI) Test battery. J. Speech Hear. Res. 21, 779-792.

Trotter,W.D., and Silverman, F.H. (1974) Does the effect of pacing speech with a miniature metronome on stuttering wear off? Percept. Mot. Skills 39 , 429-430.

van Riper, C. (1971). The Nature of Stuttering. Englewood Cliffs, NJ: Prentice-Hall, Inc.

Watkins, K. E., Smith, S. M., Davis, S. and Howell, P. (2008). Structural and functional abnormalities of the motor system in developmental stuttering. Brain 131, 50-59.

Weber-Fox, C., and Hampton, A. (2008). Stuttering and natural speech processing of semantic and syntactic constraints on verbs. J. Speech Hear. Res. 51, 1058-1071.

Weber-Fox, C., Spencer, R., Spruill, J. E. III, and Smith, A. (2004). Phonological processing in adults who stutter: electrophysiological and behavioral evidence. J. Speech Lang. Hear. Res. 47 1244-1258.

Weber-Fox, C., Spruill, J. E. III, Spencer, R., and Smith, A. (2008). Atypical neural functions underlying phonological processing and silent rehearsal in children who stutter. Dev. Sci. 11, 321-337.

Wu, J. C., Maguire, G., Riley, G., Fallon, J., LaCasse, L., Chin, S., Klein, E., Tang, C., Cadwell, S., and Lttenberg, S. (1995). A positron emission tomography $[18 \mathrm{~F}]$ deoxyglucose study of developmental stuttering. Neuroreport 6, 501-505.

Yairi, E., and Ambrose, N. G. (1999). Early childhood stuttering I: persistency and recovery rates. J. Speech Lang. Hear. Res. 42, 1097-1112.

Yamamoto, T., Maki, A., Kadoya, T. Tanikawa, Y., Yamad, Y., Okada, E. and Koizumi, H. (2002). Arranging optical fibres for the spatial resolution improvement of topographical images. Phys. Med. Biol. 47, 3429-3440.

Yamashita, Y., Maki, A., and Koizumi, H. (1996). Near-infrared topographic measurement system: imaging of absorbers localized in a scattering medium. Rev. Sci.Instrum. 67, 730-732.

Zackheim, C. T., and Conture, E. G. (2003). Childhood stuttering and speech disfluencies in relation to children's mean length of utterance: a preliminary study. J. Fluency Disord. 28, 115-141.

Zaramella, P., Freato, F., Amigoni, A., Salvadori, S., Marangoni, P., Suppjei, A., Schiavo, B., and Chiandetti, L. (2001). Brain auditory activation measured by near-infrared spectroscopy (NIRS) in neonates. Pediatr. Res. 49, 213-219.

Zimmerman, G. N. (1980). Articulatory behaviors associated with stuttering: a cineradiographic analysis. J. Speech Lang. Hear. Res. 23, 108-121.

Zocchi, L., Estenne, M., Johnston, S., Del Ferro, L., Ward, M. E., and Macklem, P. T. (1990). Respiratory muscle incoordination in stuttering speech. Am. Rev Respr. Dis. 141, 1510-1515.

Conflict of Interest Statement: The authors declare that the research was conducted in the absence of any commercial or financial relationships that could be construed as a potential conflict of interest.

Received: 14December 2010; paperpending published: 22 January 2011; accepted: 04 April 2011; published online: 27 April 2011. Citation: Sato Y, Mori K, Koizumi T, Minagawa-Kawai Y, Tanaka A, Ozawa E, Wakaba Yand Mazuka R (2011) Functional lateralization of speech processing in adults and children who stutter. Front. Psychology 2:70. doi: 10.3389/fpsyg.2011.00070 This article was submitted to Frontiers in Language Sciences, a specialty of Frontiers in Psychology.

Copyright (c) 2011 Sato, Mori, Koizumi, Minagawa-Kawai, Tanaka, Ozawa, Wakaba and Mazuka. This is an open-access article subject to a non-exclusive license between the authors and Frontiers Media SA, which permits use, distribution and reproduction in other forums, provided the original authors and source are credited and other Frontiers conditions are complied with. 